\title{
TRADICIONES TÉCNICAS Y PRODUCCIÓN CERÁMICA VIRÚ-GALLINAZO Y MOCHICA: NUEVAS MIRADAS SOBRE LAS RELACIONES ENTRE DOS GRUPOS sociales del Período Intermedio Temprano en la Costa Norte Del Perú
}

\author{
Alicia Espinosa ${ }^{\mathrm{a}}$ \\ Gabriel Prieto ${ }^{\mathrm{b}}$ \\ Walter Alva
}

\begin{abstract}
Resumen
Durante largo tiempo, los grupos Virú-Gallinazo fueron considerados como los predecesores de los mochica, aunque recientes investigaciones tienden a demostrar que coexistieron durante el Periodo Intermedio Temprano (200 a.C.-600 d.C.) en la Costa Norte del Perú. El uso sistemático de la tipología cerámica para definir las culturas pasadas explica en gran parte esta confusión. Las presentes investigaciones tienen como objetivo la definición de las fliaciones culturales entre ambos grupos, por una comparación de sus modos de producción cerámica. En esta perspectiva, usamos la tecnología cerámica para definir tradiciones técnicas asociadas a grupos sociales. Esta metodología consiste en el estudio de los procesos de elaboración de la cerámica, por análisis de huellas diagnósticas a escala macroscópica y microscópica, sobre las superficies y secciones. Esta metodología fue aplicada sobre 4212 cerámicas procediendo de cinco colecciones. Este conjunto de análisis nos llevó a determinar tres tradiciones técnicas: el martillado asociado al contexto de producción Virú-Gallinazo, y el enrollado y moldeado a la producción Mochica. Aparece que ambas poblaciones no pertenecian a la misma comunidad de filiación, aunque mantenian frecuentes contactos. La tecnología cerámica se revela entonces una perspectiva potente que supera las descripciones tipológicas y estilísticas para definir filiaciones culturales.
\end{abstract}

Palabras clave: tecnología cerámica, tradiciones técnicas, manufactura, cadena operativa, filiación cultural, cultura Virú-Gallinazo, cultura Mochica.

\footnotetext{
Abstract INTERMEDIATE PERIOD ON THE NORTHERN COAST OF PERU

a Universidad Paris 1-Panthéon Sorbonne

Correo electrónico: espinosa-alicia@hotmail.fr

b Assistant Professor, Department of Anthropology, University of Florida

Correo electrónico: ogabriel.prietob@ufl.edu

c Museo Tumbas Reales de Sipán

Correo electrónico: walteralvaarqueologo@hotmail.com
}

VIRÚ-GALLINAZO AND MOCHICA TECHNICAL TRADITION AND CERAMIC PRODUCTION: NEW PERSPECTIVES ON THE RELATIONSHIPS BETWEEN TWO SOCIAL GROUPS OF THE EARLY

For a long time, the Viru-Gallinazo populations were considered as the predecessors of the Mochicas, when recent investigations tend to show that they both coexisted during the Early Intermediate Period (200 B.C.-600 A.D.) on the 
Northern Coast of Peru. The systematic use of typology to define ancient cultures explains in great part this confusion. The research presented aim to define the cultural filiations of these two groups, by a comparison of their ceramic production modes. In this context, we use ceramic technology to define technical traditions associated to social groups. This methodology consists in the study of ceramic manufacture, by analyzing surface features at a macroscopic and microscopic scale, on the surfaces and sections. This methodology was applied on 4212 ceramics from five collections. These analyses lead to the identification of three technical traditions: the hammering associated to the Viru-Gallinazo ceramic production and the coiling and molding for the Mochica production context. It then appears that the Viru-Gallinazo and Mochica populations didn't belong to the same community of practice, but still maintained frequent contacts. Ceramic technology then represents a powerful approach to go beyond typological and stylistic descriptions in order to define cultural filiations.

Keywords: Ceramic technology, technical traditions, fashioning, operative chain, cultural affliation, Virú-Gallinazo culture, Mochica culture

\section{Introducción}

Desde la década de 1940, los arqueólogos que trabajaron en la Costa Norte del Perú consideraron a las poblaciones virú-gallinazo como las predecesoras de los mochica (100-800 d.C.), presumiendo que existía una filiación cultural directa entre ambas (Bennett 1950; Larco Hoyle 1945). No obstante, recientes investigaciones tienden a demostrar que estas dos poblaciones podrían haber coexistido hasta 600 d.C. (Millaire y Morlion 2009). Desde entonces, la redefinición del fenómeno Virú-Gallinazo se vuelve un imperativo para mejorar nuestra comprensión del paisaje sociocultural de la Costa Norte durante el Período Intermedio Temprano (200 a.C.-600 d.C.). En este marco, los nuevos proyectos arqueológicos que se emprendieron en el valle de Virú, permitieron percibir con otra perspectiva los vínculos entre los virú-gallinazo y los mochica, y sus modos de organización política (Fogel 1993: 295-297; Bourget 2010: 220-222; Millaire 2010: 6190).

Las investigaciones que se presentan aquí se inscriben en esta dinámica reciente y renovada, y buscan contribuir a caracterizar el sistema económico y social Virú-Gallinazo, a través de una definición de sus tradiciones técnicas y modos de producción cerámica. Además, el anclaje diacrónico del estudio, que consiste en la comparación de las tradiciones técnicas virú-gallinazo y mochica, conlleva a establecer el grado de filiación tecnológica entre ambos grupos. En este marco, se propone una definición del medio tecnológico durante el Período Intermedio Temprano, desde la perspectiva de las producciones cerámicas, para comprobar si ambos grupos pertenecían al mismo grupo social.

El enfoque metodológico aplicado con esta intención es el de la tecnología cerámica, una metodología basada sobre el concepto de cadena operativa, que consiste en la restitución de los métodos y técnicas de elaboración de las vasijas gracias a la observación de huellas diagnósticas (Roux 2016: 165). Los resultados propuestos se basan en el análisis de más de 4200 fragmentos y cerámicas completas procedentes de los sitios Sipán, Huaca Prieta, Pampa la Cruz, Huacas de Moche (Fig. 1), y una muestra de 49 vasijas del Museo Larco (Lima).

El presente trabajo introduce al lector a los principales conocimientos sobre las poblaciones virú-gallinazo y su relación con los grupos mochica, y presenta los fundamentos del método de la tecnología cerámica aplicada en el marco de esta investigación, así como el corpus analizado. La parte siguiente provee una descripción de las tradiciones técnicas identificadas en las colecciones, y una reflexión en torno a nuestra problemática que cuestiona las filiaciones entre las poblaciones virú-gallinazo y mochica.

\section{Antecedentes}

Los grupos virú-gallinazo y mochica se desarrollaron en la Costa Norte del Perú, una región caracterizada por un clima árido, y un paisaje desértico donde los ríos que descienden de la sierra generan 
Figura 1. Mapa de los principales sitios mencionados en el texto, ubicados en la Costa Norte del Perú, departamentos de Lambayeque y La Libertad (Edición: A. Espinosa, ASTER GDEM es un producto de METI y NASA).

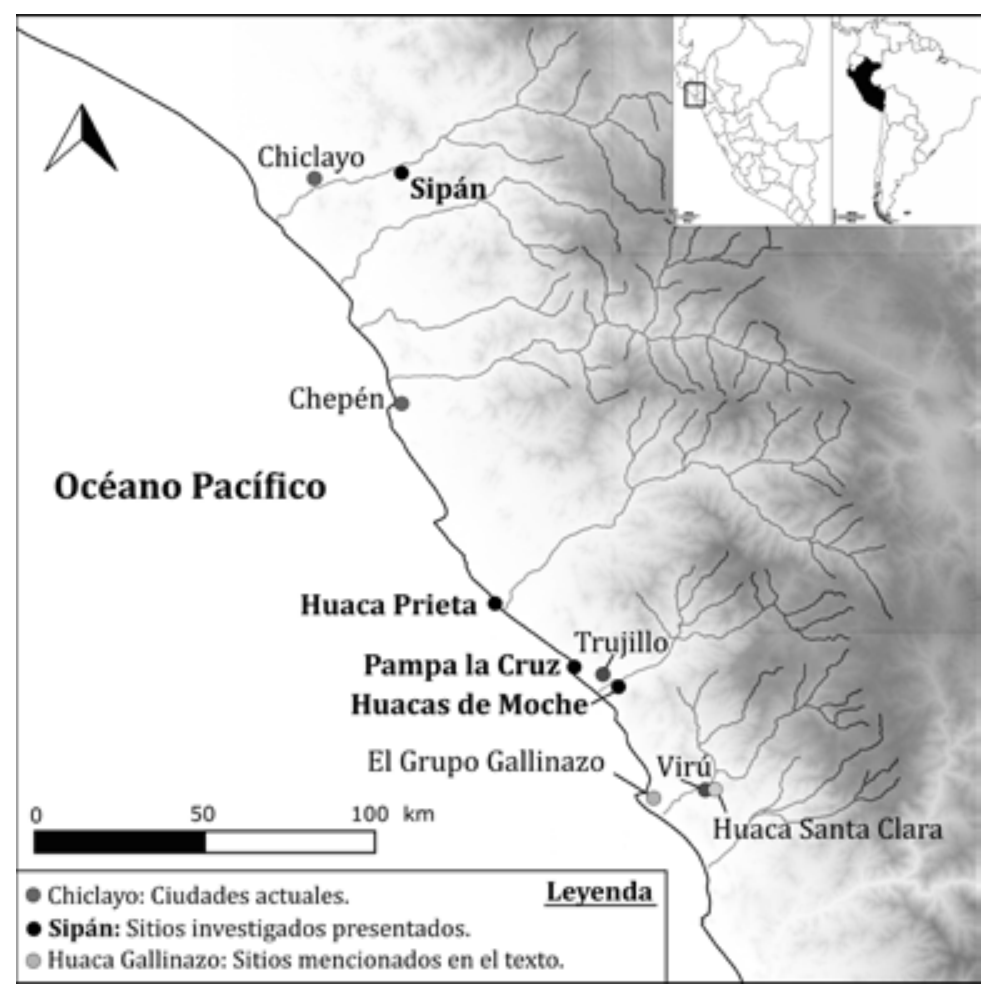

valles fluviales (Collin-Delavaud 1984: 7). Los grupos virú-gallinazo (200 a.C.-600 d.C.) se asentaron principalmente en el valle de Virú, en donde se ubica el sitio llamado el Grupo Gallinazo, uno de sus mayores centros de ocupación (Fogel 1993: 256). Por un lado, Rafael Larco Hoyle (1945: $1,11)$ y luego Wendell C. Bennett (1950) fueron los primeros en definir los rasgos culturales y la organización de la sociedad Virú-Gallinazo a partir de sus excavaciones de contextos funerarios (Collier 1955: 23; Tantaleán 2016: 124).

Tras estos trabajos pioneros, la cultura Virú-Gallinazo se volvió a definir en los años 1940 con la ocasión del Proyecto Valle de Virú. Este proyecto innovador permitió entender la secuencia ocupacional del valle de Virú, gracias a las excavaciones de Bennett (1950: 38-63), los cortes estratigráficos de Strong y Evans (1952), de Collier (1955), y las colecciones de superficie de Ford (1949). Este proyecto permitió la elaboración de una tipocronología basada en la evolución de los tipos cerámicos en estratigrafía, usada después por Willey (1953) en su estudio de los esquemas de ocupación del valle.

En su tipología cerámica, Ford (1949: 63-65) identificó cinco tipos cerámicos para el Período Gallinazo:

- El Castillo Decorado (Castillo Modelado y Castillo Inciso).

- El Gallinazo Negativo.

- El Carmelo Negativo.

- El Gallinazo Broad-Line-Incised.

La apelación Castillo Decorado, frecuentemente usada, agrupa en realidad dos tipos. El primer tipo, el Castillo Modelado, se destaca por su decoración modelada, como los cántaros cara-gollete (Fig. 2), o las aplicaciones antropomorfas, zoomorfas, y bandas aplicadas e impresas sobre las partes superiores de los cuerpos. El segundo tipo, el Castillo Inciso, se caracteriza por diseños geométricos incisos o impresos (Fig. 3). 


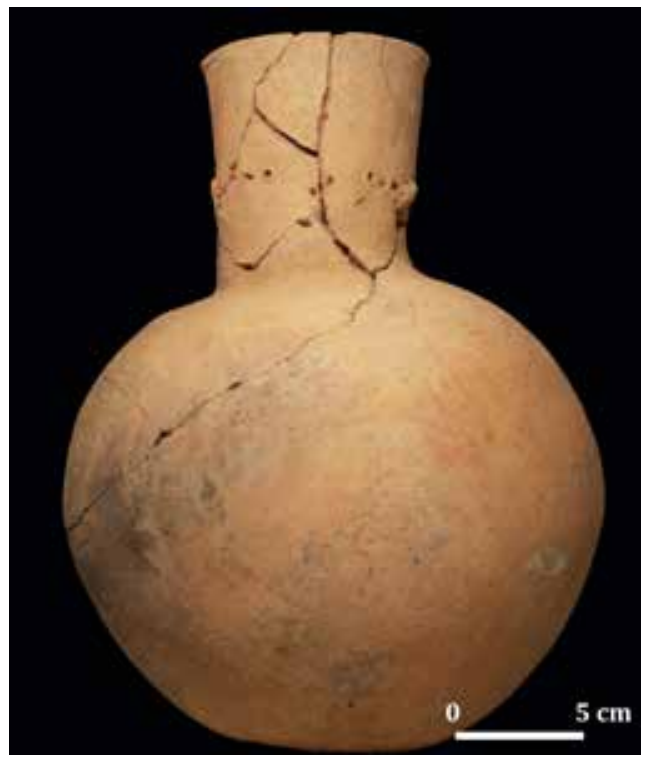

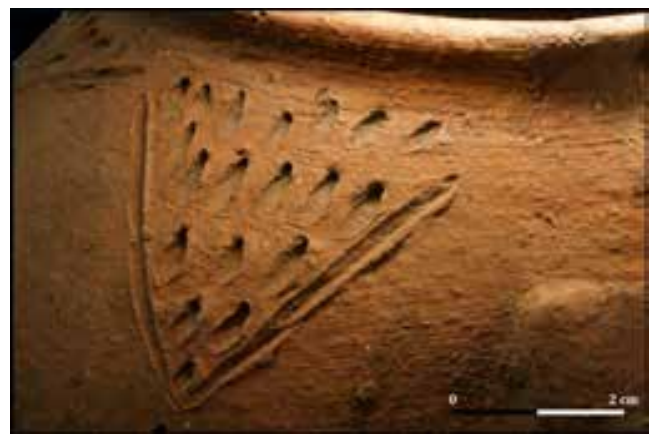

Figura 2. Izquierda: cántaro cara-gollete del tipo Castillo Modelado, Sipán, tumba 7 (Foto: A. Espinosa).

Figura 3. Derecha: olla del tipo Castillo Inciso, Museo Larco, Lima, Perú ML017061 (Foto: A. Espinosa).

Los siguientes tipos, el Gallinazo Negativo (Fig. 4) y el Carmelo Negativo (Fig. 5), se reconocen por su decoración geométrica realizada por la técnica del ahumado sobre cántaros, doble botellas, y formas escultóricas (Larco Hoyle 1945: 20; Strong y Evans 1952: 301-309). La diferencia entre ambos tipos se debe a que los diseños de las vasijas del Gallinazo Negativo tienen el color oxidado de la superficie, sobre un fondo ahumado, cuando los del Carmelo Negativo suelen tener el color del humo.

El Gallinazo-Broad-Line-Incised se distingue por sus ranuras gruesas realizadas por incisión sobre formas abiertas como los cuencos (Fig. 6). Existe además para este Período Gallinazo una serie de tipos no decorados como el Castillo llano, considerados como la cerámica doméstica usada cotidianamente (Fig. 7). Cabe mencionar que dentro de la producción virú-gallinazo se encuentran también una categoría de formas escultóricas, particularmente en contextos funerarios, y que Ford no cita (Larco Hoyle, 1945: 19; Bennett, 1950: plate 8). Según Ford (op. cit.: 64), todos estos tipos fueron producidos a lo largo del Período Gallinazo, sin cambios perceptibles.

Desde el Proyecto Valle de Virú, las poblaciones virú-gallinazo fueron entonces consideradas como una sociedad que se desarrolló principalmente en este valle epónimo, antes de ser incorporada al sistema social, cultural y político de los mochica a partir de 100 d.C. En esta perspectiva, la tipología cerámica se usó para hacer coincidir tipo y cultura en contextos arqueológicos. Sin embargo, desde aquel entonces, se acostumbró usar el Castillo Decorado como único marcador cultural Virú-Gallinazo, porque era el tipo más frecuentemente hallado en contextos. Para Donnan (2009: 30), esta práctica conllevó a lo que él denomina «la ilusión Gallinazo».

La presencia del tipo Castillo Decorado en los centros mochica fue en efecto entendida como el marcador de la presencia de los grupos virú-gallinazo, incorporados a la sociedad mochica (Shimada y Maguińa 1994: 56). Sin embargo, la frecuente y larga coexistencia de este tipo cerámico en las tumbas y áreas domésticas de los centros cívico-ceremoniales mochica, planteó numerosas interrogaciones sobre la pertinencia de tales supuestos (Chapdelaine et al. 2009: 186; Franco et al. 2009: 97-100; Uceda et al. 2009: 109-115). Era además notable constatar que los tipos negativos no se encontraron junto con cerámica mochica. En paralelo, los nuevos fechados radiocarbónicos para el valle de Virú demostraron que los grupos virú-gallinazo y mochica fueron contemporáneos hasta 600 d.C. (Millaire 2010: 6190). La pregunta consistió entonces, en saber si se podía efectivamente considerar el tipo Castillo Decorado como un marcador Virú-Gallinazo. 


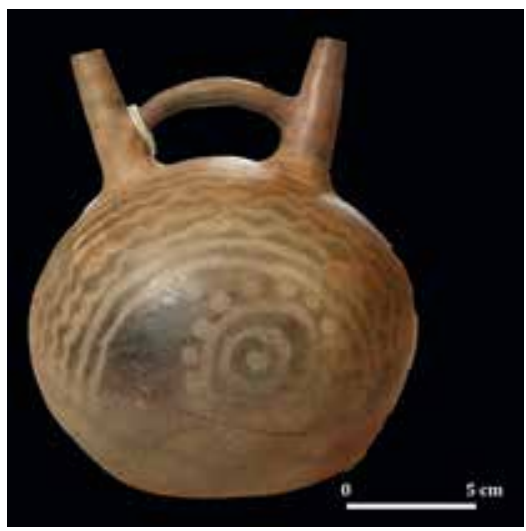

Figura 4. Botella del tipo Gallinazo Negativo, Huaca Prieta 41.2/4567 (Foto: A. Espinosa, cortesia de la Division of Anthropology, American Museum of Natural History).

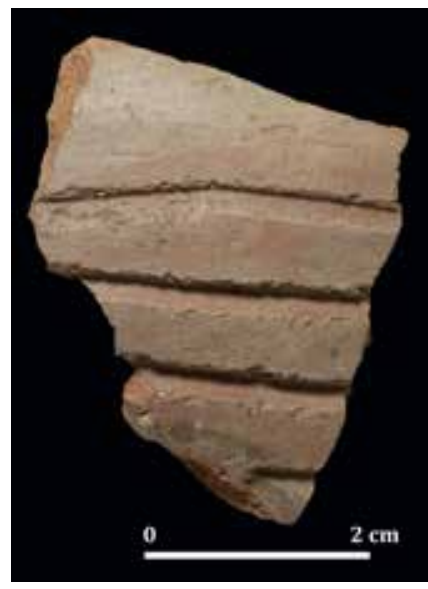

Figura 6. Fragmento del tipo Gallinazo-Broad-line incised 41.1/8565 (Foto: A. Espinosa, cortesía de la Division of Anthropology, American Museum of Natural History).

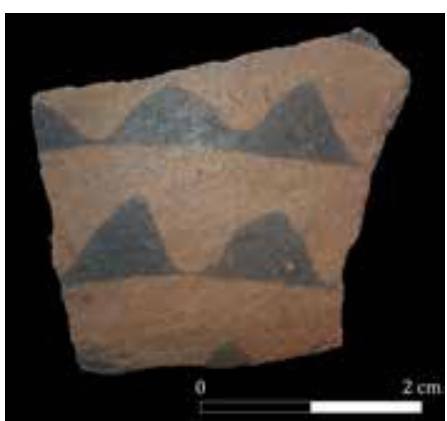

Figura 5. Fragmento de vasija Carmelo Negativo, Huaca Santa Clara (Foto: A. Espinosa).

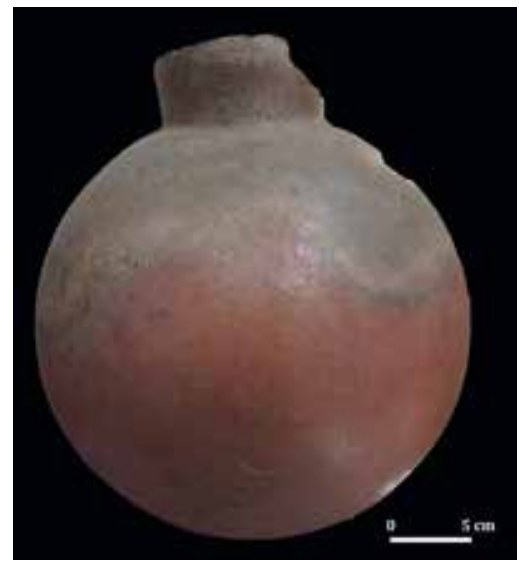

Figura 7. Cántaro no decorado, Pampa la Cruz (A4-C5-Ce56) (Foto: A. Espinosa).

Los arqueólogos reunidos durante la mesa redonda de Trujillo en 2005 propusieron un nuevo paradigma que considera el Castillo Decorado como una tradición doméstica compartida por ambos grupos o "Tradición Nor Costeña», y el tipo negativo como el marcador de la cultura Virú-Gallinazo (Millaire 2009: 12). A pesar de este esfuerzo de redefinición del fenómeno VirúGallinazo, pocas fueron las aclaraciones sobre las relaciones que pudieron mantener con los grupos mochica (v.g., Castillo 2009).

Se propone aquí estudiar los modos de producción cerámica Virú-Gallinazo y Mochica para delimitar la distribución espacial y cronológica de sus tradiciones cerámicas, y establecer su grado de filiación tecnológica. Siendo el objetivo, determinar si durante el Período Intermedio Temprano la Costa Norte fue un ambiente tecnológico homogéneo, con una tradición preponderante, o más bien un ambiente heterogéneo con tradiciones propias en cada región. Hasta entonces, estas investigaciones pretenden contribuir a mejorar los conocimientos sobre las relaciones entre los grupos virú-gallinazo y mochica, a través del prisma de la tecnología cerámica.

\section{La tecnología cerámica}

El enfoque de la tecnología cerámica, empleado en esta investigación, fue esencialmente desarrollado por la arqueología francófona, y en nuestro caso por la investigadora Valentine Roux (CNRS - 
UMR7055). Esta metodología se basa en el concepto de cadena operativa, es decir «una serie de operaciones que transforman una materia prima en un producto» (Balfet 1991: 12; Cresswell 2010: 26). El análisis tecnológico consiste en el estudio de los procesos de elaboración de las vasijas, desde la extracción de la arcilla hasta la manufactura y la cocción. El proceso de elaboración se divide en cinco etapas (Roux 2016: 64-65, 79-100, 125-145). Primero, el esbozo, es decir la elaboración de un volumen hueco que no presenta sus características geométricas finales. El conformado es la etapa que confiere al volumen sus propiedades geométricas finales. Se realiza después el acabado, que se define por técnicas de regularización, como el alisado, que modifican la capa superficial de la pasta. La siguiente etapa, los tratamientos de superficie, abarca técnicas que transforman el estado de la superficie como el bruñido o engobado. Finalmente, las cerámicas se pueden decorar con diferentes técnicas en hueco (incisión), relieve (aplicaciones), y superficie (pintura).

La observación etnográfica ha recalcado que las tradiciones técnicas son maneras de hacer, transmitidas de generación en generación dentro de un grupo social, independientemente de su naturaleza (Arnold 1981: 37; Gosselain 2002: 11-12, 24; Roux y Courty 2005: 202; Ramón 2013: 40-41). Dicha transmisión se hace mediante un aprendizaje realizado en una gran mayoría de casos a través de un tutor perteneciente al mismo grupo social que el aprendiz (Roux 2016: 19-21). Mediante mecanismos cognitivos, el aprendiz interioriza los conocimientos transmitidos, especialmente las técnicas de manufactura. Consiguientemente, estas son poco sujetas a cambio, lo cual contribuye a la estabilidad en el tiempo de las tradiciones del grupo social (Gosselain 2000: 193; Roux 2007: 165). Al contrario, las formas y decoraciones suelen adaptarse a la demanda de los consumidores, por lo que pueden fluctuar mucho más rápidamente (Gosselain 2002: 11).

El interés por las técnicas de manufactura surgió frente a las incertidumbres causadas por el uso de la tipología morfo estilística para determinar la composición social de las sociedades pasadas. Efectivamente, varios estudios etnoarqueológicos demostraron que un conjunto de formas y diseños no siempre equivale a una cultura, y que, al contrario, diversos grupos morfo estilísticos pueden equivaler a una sola cultura.

Agnès Gelbert (2003: 43-45) desarrolló sus investigaciones en el valle del río Senegal, una región en donde el análisis tipológico no identificó un conjunto homogéneo. No obstante, sus análisis tecnológicos permitieron definir dos tradiciones técnicas distintas: el modelado practicado en el valle medio, y el moldeado en el valle alto. Entendemos de esta manera que a una homogeneidad morfo-estilística aparente, corresponde una variabilidad tecnológica que demuestra la pertenencia de estas poblaciones a dos grupos sociales distintos.

Para el Ecuador, Catherine Lara (2017: 94-100) comprobó que actualmente, a pesar de la variabilidad morfo-estilística visible en las vasijas elaboradas en las diferentes comunidades de alfareros ligados al grupo étnico cañari, todas ellas son fabricadas a partir de las mismas técnicas de manufactura. El enfoque tipológico hubiera entonces llevado a aislar tradiciones distintas correspondientes a varios grupos étnicos.

Las investigaciones tecnológicas en los Andes centrales se desarrollaron recientemente, enfocándose sobre todo en análisis arqueométricos, en particular estudios petrográficos y de composición de pigmentos (Druc et al. 2001; Ghezzi 2011; Druc 2015; Del-Solar-Velarde et al. 2016: 12). Si bien varios autores ya habían señalado el interés de realizar estudios tecnológicos (Ramón 2011: 169; García 2011: 68), de momento, el análisis tecnológico empleado en esta investigación ha sido poco aplicado en Sur América (Coutet 2014: 7-10; Lara 2017). Siendo así, nuestra investigación conforma una aplicación pionera de este método en relación con las tradiciones técnicas virúgallinazo y mochica.

Desde esta perspectiva, se considera que los gestos técnicos dejan huellas que se pueden reconocer sobre los materiales arqueológicos, y que la identificación de estas huellas permite reconstituir las cadenas operativas, es decir, las tradiciones técnicas propias a grupos socioculturales. El estudio tecnológico nos lleva entonces a determinar sucesivamente tres grupos. Primero, definimos las 
entidades y grupos técnicos por identificación de los métodos y técnicas de elaboración de la cerámica. La caracterización de las cadenas operativas se hace mediante la observación de huellas diagnósticas, que se pueden atribuir a una técnica específica. Los análisis etnográficos y experimentales, realizados en el curso de estas últimas décadas, permitieron la creación de referenciales que logran una mejor identificación de los procesos de manufactura sobre materiales arqueológicos (Coutet 2014: 7; García y Calvo 2013: 143; Lara 2017: 131-150). Los rasgos diagnósticos de las técnicas son visibles a nivel macroscópico y microscópico, usando un estéreo-microscopio, sobre las paredes internas, externas y la sección de la cerámica.

Se distinguen después los grupos tecnopetrográficos para determinar la variabilidad mineral (composición y textura) de los grupos de pastas por el examen de láminas delgadas con un microscopio petrográfico, y finalmente los grupos tecno-morfo-estilísticos en los que se atribuyen formas y tipos decorativos a cada grupo técnico. El objetivo es entender la variabilidad de la producción que puede ser funcional, cuando a cada técnica corresponde una categoría morfoestilística, o cultural, siendo las mismas formas realizadas con diferentes técnicas (Roux 2016: 273).

\section{Los sitios arqueológicos y las colecciones cerámicas}

El proyecto presentado reúne las colecciones cerámicas de los sitios Sipán, Huaca Prieta, Pampa la Cruz y Huacas de Moche, implantados en tres valles de la costa norte (Tabla 1). Se incluyó también en el análisis una muestra de cuarenta y nueve cerámicas de la colección de Rafael Larco Hoyle, que procede en su mayoría de los sitios Barbacoa, Casa Grande, Santa Ana, Sausal (Chicama) y Tomabal (Virú), actualmente conservadas en el Museo Larco (Tabla 2). Los análisis emprendidos permitieron analizar más de 3867 cerámicas, de las cuales 1618 proceden de contextos virú-gallinazo, y 2249 de contextos mochica.

\subsection{Los contextos virú-gallinazo}

La definición de las técnicas y modos de producción virú-gallinazo se realizó a través del estudio de los sitios Huaca Prieta, Pampa la Cruz, y las cerámicas del Museo Larco. Huaca Prieta es un asentamiento localizado en valle de Chicama, actualmente parte del complejo Arqueológico El Brujo. Las primeras excavaciones en el sitio se realizaron entre 1946 y 1947 en el marco del Proyecto Valle de Virú. En dicha ocasión, Junius Bird se interesó en este montículo, e identificó una ocupación que remonta al Período Precerámico Tardío (Millaire et al. 2016: E6017). Sus investigaciones evidenciaron también un contexto de ocupación Virú-Gallinazo en los sondeos 1 y 3 (Bird et al. 1985: 25, 29, 271) por la presencia de botellas del tipo Gallinazo Negativo, y cántaros cara-gollete Castillo Decorado. En el marco de la presente investigación, la totalidad del material recuperado por Bird fue analizada, es decir 527 fragmentos y seis cerámicas completas.

El sitio Pampa la Cruz, ubicado en el litoral de Huanchaco en la zona norte del valle de Moche, viene siendo investigado dentro del Programa de Investigación Arqueológico Huanchaco, dirigido por Gabriel Prieto (Universidad Nacional de Trujillo) desde 2012. Este sitio cuenta con una ocupación residencial/doméstica ininterrumpida de más de 1000 años. La ocupación más temprana en Pampa la Cruz data de alrededor de 400-350 a.C. (fechas calibradas) y continúa sin interrupción hasta 650/700 d.C. (fechas calibradas). Aunque en un principio se pensó que Pampa la Cruz conoció su mayor extensión durante el Período Intermedio Temprano (Millaire et al. 2016: E6021) o Fase III (100-400 d.C. calibrado) del sitio, hoy sabemos que su mayor extensión se dio durante la Fase I (400-200 a.C. calibrado) de ocupación en el sitio que corresponde con lo que la historia cultural denomina «Salinar» en la costa norte del Perú.

Bajo esta perspectiva, la ocupación de la Fase III o Virú-Gallinazo, caracterizada por la construcción de numerosas habitaciones, plazas, junto a la presencia de inhumaciones y basureros (Prieto 
Tabla 1. Representación del número total de cerámicas analizadas en cada sitio.

\begin{tabular}{llcccc}
\hline Sitio & Valle & $\begin{array}{c}\text { Total } \\
\text { fragmentos }\end{array}$ & $\begin{array}{c}\text { Total } \\
\text { completos }\end{array}$ & $\begin{array}{c}\text { Numero mínimo } \\
\text { de individuos }\end{array}$ & Subtotal \\
\hline Sipán & Lambayeque & 93 & 109 & 177 & 202 \\
Huaca Prieta & Chicama & 527 & 6 & 298 & 533 \\
Pampa la Cruz & Moche & 1310 & 21 & 761 & 1331 \\
Huacas de Moche & Moche & 2064 & 34 & 467 & 2097 \\
\hline Total & & 3994 & 170 & 1703 & 4163 \\
\hline
\end{tabular}

Tabla 2. Representación de la procedencia y del número total de cerámicas analizadas en el Museo Larco.

\begin{tabular}{llc}
\hline Sitio & Valle & Total completo \\
\hline Tomabal & Virú & 9 \\
Indefinido & Virú & 6 \\
Barbacoa & Chicama & 1 \\
Casa Grande & Chicama & 1 \\
Santa Ana & Chicama & 5 \\
Sausal & Chicama & 2 \\
Indefinido & Chao & 3 \\
Indefinido & Santa & 1 \\
Indefinido & Indefinido & 21 \\
\hline Total & & 49 \\
\hline
\end{tabular}

2018: 98-220), es la continuación de una gran fase habitacional previa. En los contextos virúgallinazo o de la Fase III se reveló la presencia de más de 1310 tiestos y 21 vasijas enteras que ofrecen una visión amplia de diferentes tipos decorados (Gallinazo Negativo, Castillo Decorado, GallinazoBroad-Line Incised) y no decorados del estilo Virú-Gallinazo.

\subsection{Los contextos mochica}

La caracterización de la producción mochica se realizó gracias al estudio de los materiales de los Complejos Arqueológicos Sipán y Huacas de Moche. En estos asentamientos, los contextos se eligieron en función de la presencia de cerámica Castillo Decorado, previamente asociada a una ocupación Virú-Gallinazo.

El complejo Sipán, implantado en el valle de Lambayeque, es conocido por sus dos pirámides de adobe, asociadas a una plataforma funeraria donde se halló la tumba del Señor de Sipán, que están investigadas desde 1987 (Alva y Donnan, 1993: 27, 43). El sitio fue ocupado mayormente por la sociedad Mochica, desde el Mochica Temprano (100-200/250 d.C.). Los contextos analizados proceden de la plataforma funeraria, lo que incluye la tumba 7 del Guardián (Chero Zurita, 2015: 3). Para el sitio, se analizó un total de 93 fragmentos y 109 cerámicas completas.

El complejo Huacas de Moche, ubicado en la parte baja del valle de Moche, fue ocupado entre 100 y 850-900 d.C. El sitio se caracteriza por la presencia de la Huaca del Sol y de la Luna, entre 
las cuales se extendió una zona urbana donde se edificaron varios conjuntos arquitectónicos, plataformas, sectores residenciales, a veces asociados a talleres (Uceda 2016: 69). El Proyecto Arqueológico Huaca de La Luna, que fue dirigido desde 1991 por Santiago Uceda y Ricardo Morales, brindó mucha información sobre una posible coexistencia entre comunidades virú-gallinazo y mochica. Nuestro interés se centró en los contextos en donde se evidenció la presencia de cerámica Castillo Decorado junto al Mochica Decorado, lo que incluye las tumbas de la Plataforma Uhle (Chauchat et al. 2009: 95), los contextos sacrificiales de la Plaza 3C, y el Complejo Arquitectónico 35 de la zona urbana (Uceda et al. 2009: 112-115). En total, pudimos observar más de 2064 tiestos y 34 cerámicas completas.

\section{Resultados}

El análisis de los contextos virú-gallinazo y mochica nos permitió destacar tres tradiciones técnicas que presentamos a través de sus huellas diagnosticas identificadas según una escala macroscópica y microscópica. En esta presentación seguimos el orden de las acciones de la cadena operativa, y nos concentramos en la descripción de las etapas de la cadena operativa que son las más interiorizadas por los alfareros durante el aprendizaje, es decir la manufactura, que permiten definir la comunidad de filiación de los alfareros (Gosselain 2000: 192-193; Gallay 2011: 326-327). De esta manera, describimos aquí las técnicas del esbozo y conformado. Siendo los análisis petrográficos todavía en curso, no discutiremos en esta ocasión sus resultados.

\subsection{Grupo técnico 1: el martillado}

\section{La manufactura}

El martillado es una técnica que implica el uso de una herramienta activa que sirve para golpear una masa de arcilla homogénea contra un soporte (herramienta pasiva). Aunque el martillado y el paleteado sean dos técnicas vinculadas a la percusión, el paleteado se distingue por la percusión simultánea de las paredes internas y externas con dos herramientas activas (Roux 2016: 96; Lara 2017: 96-98). En el caso de una percusión de escasa intensidad, el paleteado se puede ejercer sin soporte, mientras que el uso de este último para el martillado es sistemático.

La técnica del martillado se caracteriza por los siguientes rasgos diagnósticos sobre las cerámicas: a escala macroscópica, se observa una topografía externa homogénea y un perfil regular de arriba hacia abajo, con un espesor medio de 7 milímetros para el conjunto estudiado. Este control de la topografía y del grosor de las paredes sugiere el uso de un soporte durante el martillado. Las superficies internas son atravesadas por concavidades de percusión, correspondientes a la impronta del golpeador (Fig. 8). A escala microscópica, la microtopografía es alternativamente alisada fluidificada e irregular, asociada a crestas de barbotina y micro arrancamientos (Fig. 9). Este conjunto de rasgos indica una percusión sobre una pasta húmeda, con el uso de una herramienta mojada (Roux 2016: 214; Lara 2017: 136-137). En la sección de los fragmentos, se pueden apreciar fisuras alargadas, subparalelas a la superficie, así como una porosidad asociadas a un gesto de percusión (Fig. 10).

Los cuellos de las vasijas martilladas se realizaban añadiendo rollos de pasta sobre la parte superior del cuerpo, en posición oblicua interna. A escala macroscópica, el enrollado se manifiesta por numerosas fisuras que indican la unión entre dos rollos, resaltes (Fig. 11), y fracturas preferenciales entre el cuerpo y el cuello. A escala microscópica, observamos poros de orientación subcircular en sección. Se daba la forma final al cuello por presiones continuas con la mano mojada. Esta técnica se manifiesta por una serie de estrías concéntricas de borde nervado localizadas específicamente sobre el borde y el punto de unión entre el cuerpo y el cuello, y estrías reticuladas correspondientes al uso de la mano (Roux 2016: 237; fig. 12). 


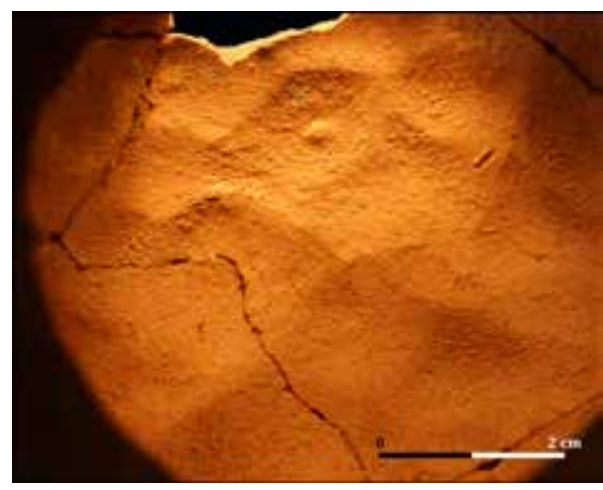

Figura 8. El martillado: concavidades de percusión sobre la base de una botella, Pampa la Cruz (PLC126-A3-RC2-Ce03) (Foto: A. Espinosa).

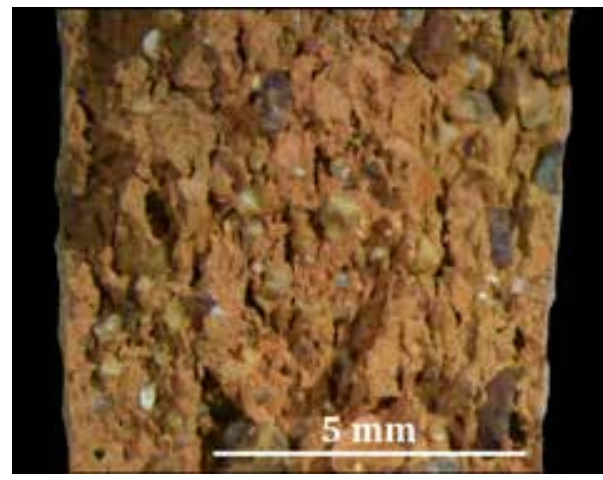

Figura 10. El martillado: fisuras alargadas subparalelas a la superficie, Sipán (Foto: A. Espinosa).

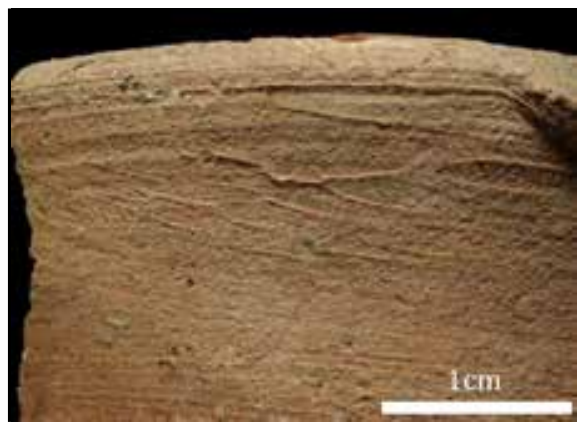

Figura 12. Arriba. El borde: estrías nervadas y reticuladas, Pampa la Cruz (Foto: A. Espinosa).

Figura 13. Derecha. La técnica del enrollado: ejemplo de fisura, Pampa la Cruz (PLC-A3-RC2-FC84-2) (Foto: A. Espinosa).

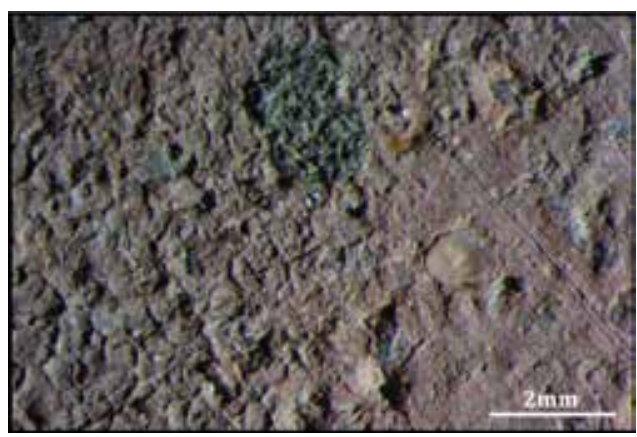

Figura 9. El martillado: crestas de barbotina y microarrancamientos. Pampa la Cruz (PLC-A3-C3-FC91-2) (Foto: A. Espinosa).

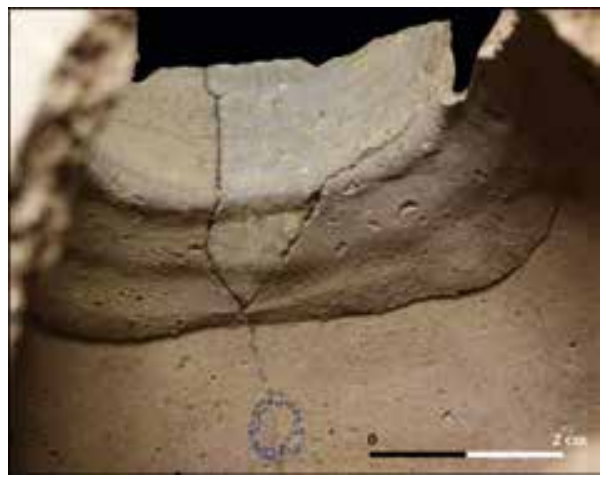

Figura 11. El borde: resalte concéntrico. Huaca Prieta. 41.2/4732 (Foto: A. Espinosa, cortesia de la Division of Anthropology, American Museum of Natural History).

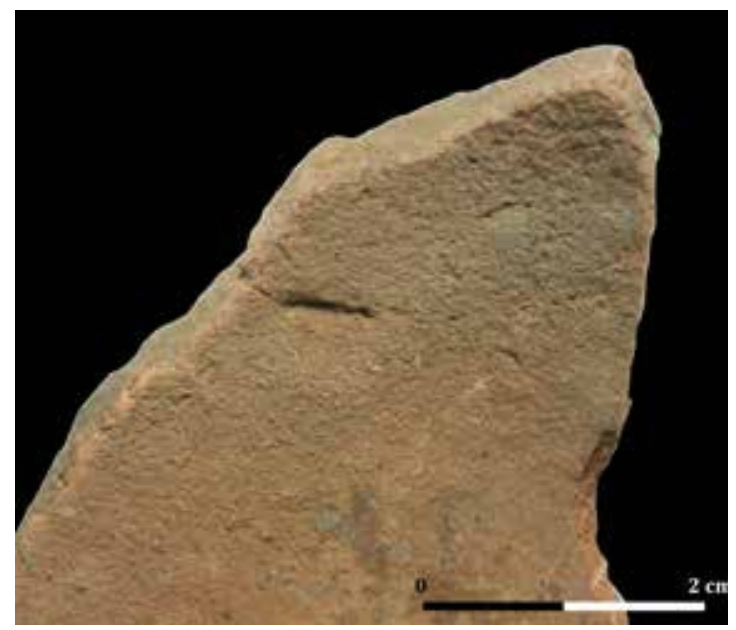



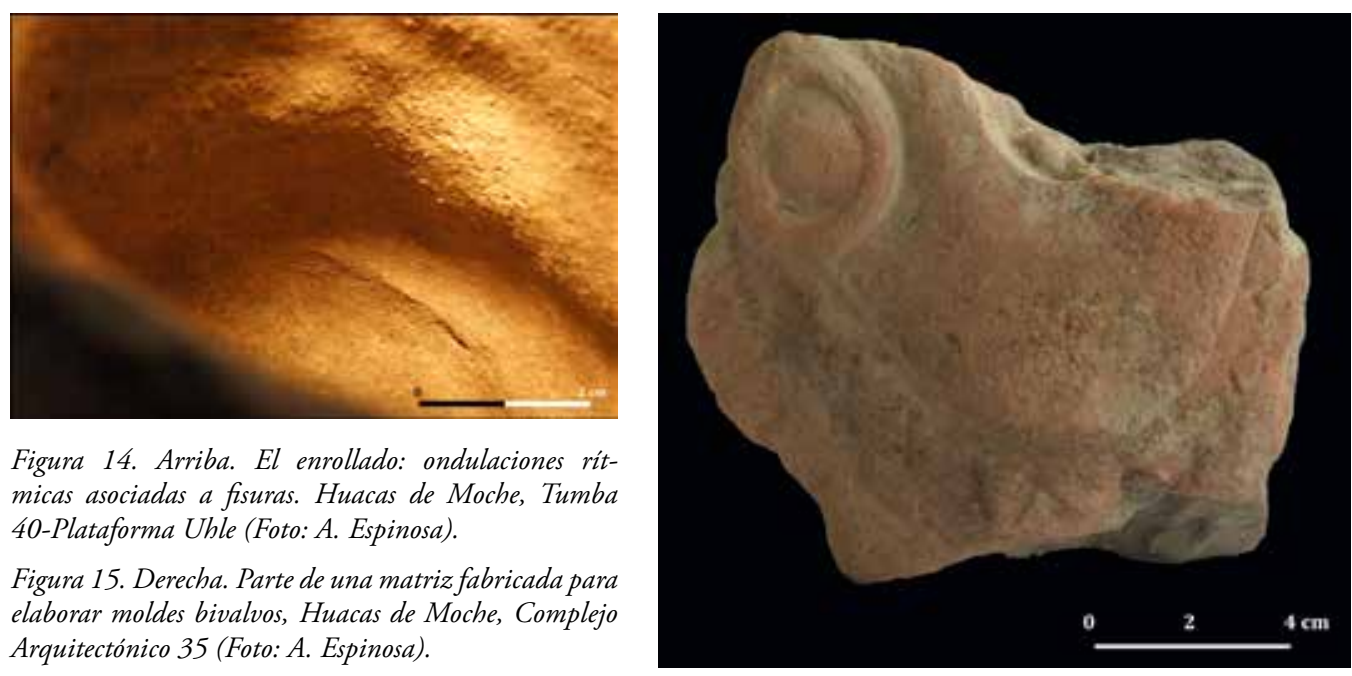

Figura 14. Arriba. El enrollado: ondulaciones ritmicas asociadas a fisuras. Huacas de Moche, Tumba 40-Plataforma Uhle (Foto: A. Espinosa).

Figura 15. Derecha. Parte de una matriz fabricada para elaborar moldes bivalvos, Huacas de Moche, Complejo Arquitectónico 35 (Foto: A. Espinosa).

\section{Formas y decoraciones}

El martillado es una técnica que se empleaba para realizar una gama de formas que incluye cancheros, cántaros, botellas, doble-botellas silbadoras, ollas con cuello y tinajas. La mayoría de las vasijas no presentan ningún tipo decoración. Sin embargo, las técnicas empleadas para decorar las demás vasijas son muy variadas. Distinguimos al respeto decoraciones en hueco (incisión, impresión y perforación), en relieve (aplicación), en superficie (el negativo). Las decoraciones más representativas son aquellas realizadas sobre los cántaros. Una primera variante consiste en adornar los cuellos con aplicaciones, resaltadas con incisiones e/o impresiones, para formar diseños antropomorfos o zoomorfos. La segunda variante incluye las bandas aplicadas e impresas sobre la parte superior de los cuerpos. Otra técnica decorativa frecuentemente usada es el negativo, para crear diseños geométricos sobre las botellas, doble-botellas silbadoras, y una tinaja en Pampa la Cruz.

\subsection{Grupo técnico 2: el enrollado}

\section{La manufactura}

El enrollado es una técnica que consiste en elaborar recipientes mediante la superposición de rollos de pasta. En el caso presente los cuerpos y los cuellos de las vasijas se concebían disponiendo los rollos en posición oblicua sobre una tortilla modelada. Los rasgos diagnósticos que permitieron identificar esta técnica son similares a aquellos presentados para la tradición del martillado en cuanto a la manufactura de los cuellos.

A escala macroscópica, además de las fisuras (Fig. 13), resaltes y fracturas preferenciales, notamos lo siguiente. Primero, un perfil irregular y una topografía discontinua con ondulaciones rítmicas (Fig. 14). A escala microscópica, se aprecian en sección vacíos que indican la unión entre dos rollos, y una orientación subcircular de la microestructura. En lo que se refiere al conformado, la superficie se regularizaba por presiones discontinuas, dejando una serie de depresiones e improntas digitales en superficie interna y externa.

\section{Formas y decoraciones}

Como para el caso del martillado, la técnica del enrollado fue empleada en su mayoría para realizar cerámicas no decoradas como los cuencos, cántaros, tinajas y ollas. Sin embargo, notamos para algunos casos la presencia de decoraciones antropomorfas o zoomorfas sobre los golletes de los cántaros, que ya hemos mencionado en el caso del martillado. 


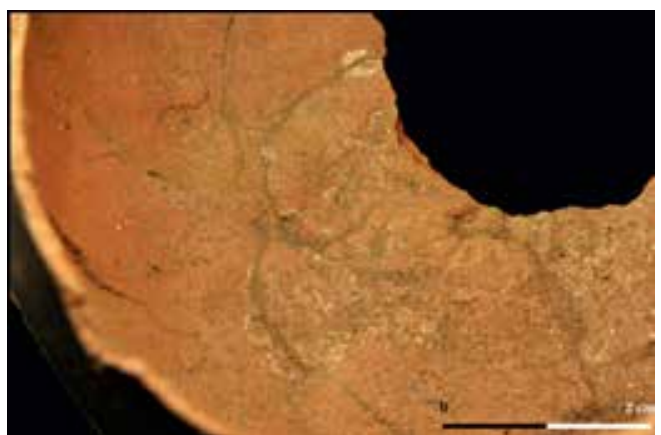

Figura 16. El moldeado: vasija sellada por un rollo de pasta en espiral. Museo Larco, Lima, Perú ML016477 (Foto: A. Espinosa).

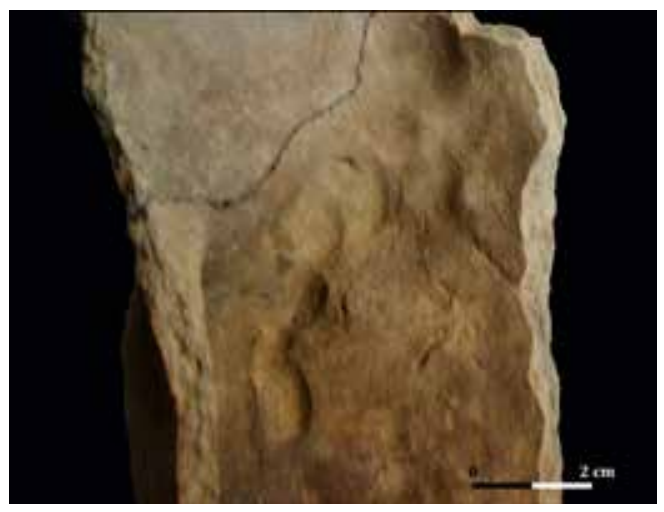

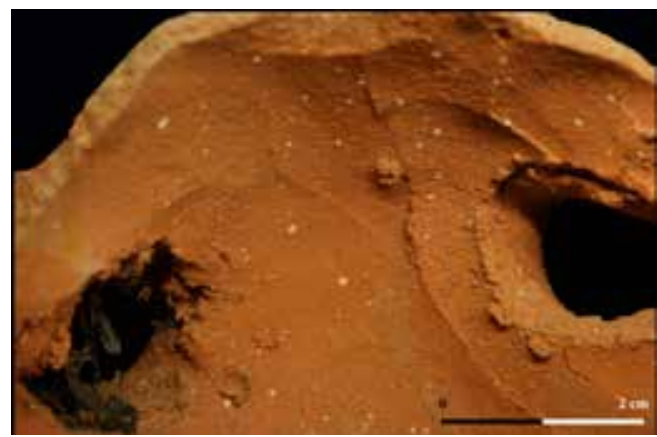

Figura 17. El moldeado: desplazamientos de pasta en superficie, Museo Larco, Lima, Perú. ML016477 (Foto: A. Espinosa).

Figura 18. El moldeado: numerosas improntas de dedos y uñas en superficie interna, Huacas de Moche, Plaza 3C (Foto: A. Espinosa).

\subsection{Grupo técnico 3: el moldeado}

\section{La manufactura}

La técnica del moldeado consiste en rellenar un molde con las manos para realizar el cuerpo de las vasijas. El primer paso del moldeado consiste en la elaboración de una matriz, modelando una masa de arcilla a partir de la cual se creaba el molde, compuesto de dos partes (Donnan 2004: 22-25; fig. 15). Después, se estiraban pedazos de arcilla contra la pared, insistiendo en las partes con más detalles decorativos como los rostros. Una vez formado el cuerpo, la base se sellaba con un rollo de pasta en espiral, aunque en algunos casos se usaba una tortilla modelada (Donnan 2004: 26-27; fig. 16). Los apéndices como los golletes y asas se realizaban con rollos de pasta aplicados mediante el sistema de espiga y mortaja.

A escala macroscópica, el moldeado se caracteriza por una topografía externa homogénea debido al uso de un soporte, un perfil regular con un espesor medio de 0.4 centímetros de arriba hacia abajo para el conjunto analizado. Se observan además desplazamientos de pasta en superficie interna que resultan del estiramiento de la pasta contra el molde (Fig. 17) e improntas de dedos y uñas (Fig. 18). A escala microscópica, notamos una microtopografía fluida e irregular, asociada a estrías de borde filiforme, organizadas horizontalmente, dejadas por el paso de la mano sobre una superficie húmeda. En sección se aprecian fisuras alargadas subparalelas a la superficie. Valga recalcar al respecto, que, según experimentaciones, el aplanamiento de los poros es más importante en caso de martillado, debido a una percusión más fuerte (Roux 2016: 212). Una gran mayoría de las cerámicas moldeadas fueron realizadas con un molde bivalvo. Sin embargo, para ciertas vasijas se necesitan todavía más datos comparativos para poder definir los tipos de moldes usados. 


\section{Formas y decoraciones}

El moldeado es una técnica que permitía realizar específicamente formas decoradas, en particular botellas asa-estribo con representaciones escultóricas (Gayoso 2016: 291-292). Las cerámicas realizadas con moldes bivalvos representan en su mayoría personajes antropomorfos, resaltados con pintura. Para las demás cerámicas, solo se pudo identificar una botella fitomorfa en el Museo Larco (ML016477).

\section{Interpretaciones de los resultados y discusión}

El análisis de las colecciones cerámicas procedentes de contextos virú-gallinazo y mochica permitió destacar tres tradiciones técnicas predominantes: el martillado, el enrollado y el moldeado. En los asentamientos virú-gallinazo identificamos estas tres tradiciones técnicas. La tradición por martillado es predominante en estos contextos, en específico en el sitio Pampa la Cruz, en donde representa el 99.4\% (NR: 1088) de la muestra del estudio. La manifestación muy escasa de la técnica del enrollado en las colecciones nos lleva a considerarla como una tradición anecdótica, que, siguiendo con el ejemplo de Pampa la Cruz, representa el 0.3\% (NR: 3) de la muestra. Lamentablemente, esta técnica solo se identificó sobre los bordes y cuerpos superiores de formas abiertas de tipo cuenco y plato. Como consecuencia, se necesitará más material comparativo, correspondiente a los cuerpos inferiores y bases, para reconstituir sus procesos de elaboración. Una posibilidad es que solo los cuerpos superiores y cuellos se realizaron por enrollado, siendo las bases y cuerpos inferiores confeccionados por martillado. La técnica de manufactura por moldeado es también un fenómeno anecdótico en contexto de producción virú-gallinazo, que representa el $0.3 \%$ de las cerámicas en Pampa la Cruz (NR: 4). Esta técnica se empleaba además para realizar una categoría morfoestilística especifica: las vasijas escultóricas.

Finalmente, al observar la variabilidad tecnológica de la producción virú-gallinazo, constatamos que los alfareros adaptaban sus maneras de hacer según el tipo de recipiente. Los cántaros, tinajas y ollas se elaboraban con la técnica del martillado, los platos y cuencos por enrollado, y la técnica del moldeado se usaba para las formas escultóricas. Podemos entonces apreciar una variabilidad tecnológica funcional y no cultural. La tipología que James Ford había creado en 1949 para el Período Gallinazo, había conducido a separar distintos tipos como el Castillo Decorado y el Negativo, que hasta la fecha se consideran como la manifestación de dos fenómenos sociales diferentes. Proponemos entonces que, al contrario, la misma comunidad alfarera realizó tanto las producciones decoradas como no decoradas, sin que se trate necesariamente de diferentes unidades de producción. Hasta entonces, para la producción virú-gallinazo no podemos, por ejemplo, definir que existían dos clases de alfareros, dedicados a las vasijas decorativas y llanas. Aparece en nuestro caso que, a un conjunto variado de formas y decoraciones, corresponde una misma comunidad alfarera.

Las cadenas operativas registradas en los sitios virú-gallinazo se diferencian de aquellas identificadas en contextos mochica, en donde se determinó también la presencia de estas tres tradiciones técnicas. La tradición por moldeado es predominante en Sipán y Huacas de Moche. No obstante, los alfareros mochicas usaron un soporte que no se identificó en contexto virú-gallinazo: el molde bivalvo. El enrollado se empleaba para la producción de vasijas de cerámica no decorada como las tinajas, ollas y cuencos. Por entonces, la variabilidad que se observa dentro de la producción mochica tiene también un carácter funcional, ya que las técnicas del moldeado y enrollado se asocian a gamas de formas distintas (Fig. 18). A pesar de estas variabilidades, se puede proponer que los alfareros mochicas pertenecían a la misma comunidad de práctica, y que adaptaban sus técnicas al tipo de forma por realizar. 


\section{Conclusión}

Los resultados que se presentan permiten aclarar nuestra problemática inicial que apunta a la definición cultural, social y económica de las poblaciones virú-gallinazo, y de sus posibles filiaciones con los mochica. Esta problemática fue abordada a través del prisma de la tecnología cerámica, para definir las tradiciones técnicas y los modos de producción cerámica de ambas poblaciones. Nuestras hipótesis son sustentadas por la representatividad geográfica, cronológica y cultural de nuestra muestra de estudio. Las discusiones son el resultado de observaciones preliminares, que están actualmente profundizadas con estudios comparativos de los modos de producción cerámica en otros asentamientos mochica y virú-gallinazo.

Desde una perspectiva macrorregional, el entorno técnico de la Costa Norte durante el Período Intermedio Temprano es heterogéneo. Existían en esa época dos tradiciones técnicas predominantes vinculadas a la presencia de dos grupos sociales: el martillado asociado a las poblaciones virú-gallinazo, el moldeado y el enrollado a los mochica. En el estado actual de esta investigación, se puede decir que ambos grupos no pertenecían a la misma comunidad de práctica, y que los grupos virú-gallinazo y mochica tenían sus propios grupos de alfareros. Estas interpretaciones deberán ser confrontadas a otros tipos de producción artesanal Virú-Gallinazo y Mochica (textiles, metalurgia, entre otros), así como la arquitectura y los patrones funerarios.

La tecnología cerámica es una perspectiva potente para definir la composición de los grupos sociales pasados y sus grados de filiación, lo que permite adquirir una lectura antropológica de los ensamblajes cerámicos que va más allá de meras descripciones tipológicas y estilísticas. Los análisis tipológicos llevan a crear categorías aisladas, utilizadas como marcadores cronoculturales, al igual que los tipos Gallinazo Negativo y Castillo Decorado. Por lo tanto, el uso de esta tipología condujo a la "ilusión Gallinazo», y se consideró que el Castillo Decorado era solamente una tradición doméstica compartida por los mochicas y virú-gallinazo. Desde entonces, el estudio tecnológico nos permite considerar el fenómeno Virú-Gallinazo de manera más compleja y dinámica. Demostramos que los diferentes alfareros virú-gallinazo en la Costa Norte pertenecían a la misma comunidad de aprendizaje, y que no tienen una filiación directa con los alfareros mochica.

\section{Agradecimientos}

Ante todo, queremos agradecer Isabelle Druc y Jalh Dulanto, por darnos la oportunidad de presentar nuestros resultados durante este simposio que reunió investigadores de diferentes horizontes y países, y por permitir la edición de los artículos. Agradecemos también a Karla Patroni por la coordinación de este simposio. El proyecto de investigación, desarrollado entre 2016 al 2018, permitió fortalecer colaboraciones en diferentes instituciones del Perú, de Francia y Estados-Unidos. Expresamos nuestros sinceros agradecimientos a la Universidad Paris 1 - Panthéon Sorbonne, y al laboratorio Arqueología de las Américas (CNRS - UMR 8096) para su apoyo financiero y científico. Estamos particularmente agradecidos por la supervisión y el apoyo de Véronique Darras, Nicolas Goepfert, y Valentine Roux. Agradecemos al Instituto Francés de Estudios Andinos y su directora Evelyne Mesclier, por la oportunidad que nos ofrecieron de desarrollar nuestras investigaciones gracias a la beca Carlos Brignardello. Al Museo de Historia Natural de Nueva York por permitir el análisis de las colecciones de Huaca Prieta, especialmente gracias a la beca de estudios de colecciones, y el apoyo de Charles Spencer y Sumru Aricanli durante los análisis en el museo. Al Museo Larco, en especial a Isabel Collazos por recibirnos y hacernos conocer esta amplia colección. En el Museo Huacas del Valle de Moche-Santiago Uceda Castillo, siempre estaremos agradecidos a Santiago Uceda por recibirnos más de cuatro meses en su institución. A los profesores y estudiantes de la Universidad Nacional de Trujillo, en específico los miembros del Proyecto Arqueológico Huanchaco. A los miembros del Museo Tumbas Reales de Sipán, en especial Edgar Bracamonte por su apoyo constante. A Catherine Lara por sus consejos durante los estudios de colecciones, y por su 
ayuda en la redacción del artículo. A Téo Gaillot por su apoyo durante la edición de las fotografías. Finalmente, un gran agradecimiento a la magíster Belkys Gutiérrez y al profesor Segundo Vásquez, quienes con su ayuda y consejos contribuyeron en gran parte a la realización de este proyecto.

\section{Referencias}

Alva, W. y C. Donnan

1993 Tumbas Reales de Sipán, Fowler Museum of Cultural History, University of California, Los Angeles.

Arnold, D.

1981 A model for the identification of non-local ceramic distribution: a view from the present, en: $\mathrm{H}$. Howard y E. Morris (eds.), Production and distribution: a ceramic viewpoint, 31-44, BAR (British Archaeological Reports) International Series 120, Oxford.

Balfet, $\mathrm{H}$.

1991 Des chaînes opératoires, pour quoi faire?, en: Balfet H. (ed.), Observer l'action technique, des chaînes opératoires pour quoi faire?, 11-20, CNRS (Centre National de la Recherche Scientifique) Éditions, Paris.

Bennett, W.

1950 The Gallinazo Group, Virú Valley, Peru, Yale University Publications in Anthropology 43, Yale University Press, New Haven.

Bird, J. y J. Hyslop

1985 The Preceramic excavation at the Huaca Prieta Chicama valley, Peru, Anthropological Papers of the American Museum of Natural History, vol. 62: Parte 1, New York.

\section{Bourget, $S$.}

2010 Cultural assignations during the Early Intermediate Period, The case of Huancaco, Virú, Valley, en: J. Quilter y L.J. Castillo (eds.), New perspectives on Moche political organization, 201-222, Dumbarton Oaks Research Library and Collection, Washington, D.C.

Castillo, L.J.

2009 Gallinazo, Vicus, and Moche in the Development of Complex Societies along the North Coast of Peru, en: J.F. Millaire y M. Morlion (eds.), Gallinazo. An Early Cultural Tradition on the Peruvian North Coast, 223-231, Cotsen Institute of Archaeology Press, University of California, Los Angeles. https://doi. org/10.2307/j.ctvdjrqbs.21

\section{Chapdelaine, C., V. Pimentel y J. Gamboa}

2009 Gallinazo cultural identity in the lower Santa valley, Ceramics, architecture, burial patterns, and sociopolitical organization, en: J-F. Millaire y M. Morlion (eds.), Gallinazo. An early cultural tradition on the Peruvian North Coast, 181-206, Cotsen Institute of Archaeological Press, University of California, Los Angeles. https://doi.org/10.2307/j.ctvdjrqbs.18

Chauchat, C., B. Gutiérrez, D. Deverly, N. Goepfert y J.B. Huchet

2009 La Plataforma Uhle en Moche: una síntesis de los descubrimientos, Revista del Museo de Arqueología, Antropologia e Historia 11, 85-110.

Chero, L.

2015 Nuevos aportes en la investigación arqueológica de Sipán, Museo de sitio Huaca Rajada-Sipán, Ministerio de la Cultural del Perú, Unidad Ejecutora 005, Naylamp-Lambayeque, Chiclayo.

Collier, D.

1955 Cultural chronology and change as reflected in the ceramics of the Virú valley, Peru, Fieldiana Anthropology 43, Chicago Natural History Museum. https://doi.org/10.5962/bhl.title.6576

Collin, D. C.

1984 Las regiones costeñas del Perú septentrional. Ocupación humana, desarrollo regional, Fondo Editorial PUCP, Lima. 
Coutet, C.

2014 La caractérisation techno-stylistique de la céramique de tradition Arauquinoide en Guyane: Une approche ethnoarchéologique de la céramique amérindienne, Karapa 3, Revue d'anthroplogie des sociétés amérdindiennes anciennes, d'histoire et d'archéologie coloniale du bassin amazonien et du plateau des Guyanes, 6-20.

Cresswell, R.

2010 «Techniques et culture: les bases d'un programme de travail», Techniques \& Culture, https://doi. org/10.4000/tc.4979

Del-Solar-Velarde, N., S. Kinis, R. Chapoulie, R. Joannes-Boyau y L.J Castillo

2016 Characterization of pre-Columbian artefacts in situ through handheld portable X-ray fluorescence spectrometry: the case of ceramics from the Mochica site of San José de Moro (Peru), Heritage Science 4 (37), 1-13. https://doi.org/10.1186/s40494-016-0109-y

Donnan, C.

2004 Moche portraits from Ancient Peru, University of Texas Press, Austin.

2009 The Gallinazo illusion, en: J-F Millaire y M. Morlion (eds.), Gallinazo. An early cultural tradition on the peruvian North Coast, 17-32, Cotsen Institute of Archaeological Press, University of California, Los Angeles. https://doi.org/10.2307/j.ctvdjrqbs.9

Druc, I.

2015 Ceramic Analysis in the Andes, Deep University Press, Blue Mounds, WI.

Druc, I., R. Burger, R. Zamojska y P. Magny

2001 Ancón and Garagay Ceramic Production at the Time of Chavin de Huántar, Journal of Archaeological Science 28 (1), 29-43. https://doi.org/10.1006/jasc.1999.0587

Fogel, H.

1993 Settlements in time: a study of social and political development during the Gallinazo occupation of the north coast of Peru, tesis doctoral, Departamento de Antropología, Yale University, New Haven.

Ford, J.

1949 Cultural dating of Prehistoric sites in Virú Valley, Peru, en: Ford J. y G. Willey (eds.), Surface survey of the Virú Valley, Peru, 30-78, Anthropological Papers of the American Museum of Natural History 43, Parte $1-2$.

Franco, R. y C. Gálvez

2009 Gallinazo-style ceramics in Early Moche contexts at the el Brujo Complex, Chicama valley, en: Millaire J-F. y M. Morlion (eds.), Gallinazo. An early cultural tradition on the Peruvian North Coast, 91-104, Cotsen Institute of Archaeological Press, University of California, Los Angeles. https://doi.org/10.2307/j. ctvdjrqbs. 13

Gallay, A.

2011 Pour une ethnoarchéologie théorique, Édition Errances, Paris.

García, J.

2011 Modelado, aprendizaje y espacio social: una reflexión desde la tecnología cerámica, Revista Werkén 14, 63-74.

García. J. y M. Calvo

2013 Making pots. El modelado de la cerámica y su potencial interpretativo, BAR (British Archaeological Reports), International Series 2540, Oxford.

Gayoso, $\mathrm{H}$.

2016 Los maestros del barro: cerámica y ceramistas de la ciudad de las Huacas del Sol y de la Luna, en: S. Uceda, R. Morales y C. Rengifo (eds.), Proyecto Arqueológico Huacas del Soly de la Luna, Investigaciones en la Huaca de la Luna 2015, 289-316, Patronato Huacas del Valle de Moche, Facultad de Ciencias Sociales, Universidad Nacional de Trujillo, Trujillo.

Gelbert, A.

2003 Traditions céramiques et emprunts techniques dans la vallée du fleuve Sénégal, ed. de la Maison des sciences de l'Homme, Epistèmes, Paris. 
Ghezzi, I.

2011 El análisis composicional en el estudio de la producción y distribución de la cerámica prehispánica, Bulletin de l'Institut Français d'Études Andines 40 (1), 1-29. https://doi.org/10.4000/bifea.1565

Gosselain, O.

2000 Materializing identities: An African perspective, Journal of Archaeological Method and Theory 7 (3), 187217. https://doi.org/10.1023/A:1026558503986

2002 Poteries du Cameroun meridional. Styles techniques et rapports à l'identité, Collection de Recherches Archéologiques (CRA-Monographies), CNRS Éditions, Paris.

Lara, C.

2017 Aportes del enfoque tecnológico a la arqueología precolombina: pasado y presente de la alfarería en el valle del rio cuyes y su región (Andes sur-orientales del Ecuador), Paris Monograph in Archaeology 47, Archaeopress Publishing LTD, Oxford.

Larco, R.

1945 La cultura Virú, Sociedad geográfica americana, Buenos Aires.

Millaire. J-F.

2009 Gallinazo and the tradición norcosteña, en: Millaire J-F. y M. Morlion (eds.), Gallinazo. An early cultural tradition on the Peruvian North Coast, 1-16, UCLA, Cotsen Institute of Archaeological Press, University of California, Los Angeles. https://doi.org/10.2307/j.ctvdjrqbs.8

2010 Primary State formation in the Virú valley, North Coast of Peru, PNAS 107 (14), 6186-6191. https:// doi.org/10.1073/pnas.0911226107

Millaire, J-F. y M. Morlion (eds.)

2009 Gallinazo. An early cultural tradition on the peruvian North Coast, Cotsen Institute of Archaeological Press, University of California, Los Angeles. https://doi.org/10.2307/j.ctvdjrqbs

Millaire, J-F., G. Prieto, F. Surette, E. Redmond y C. Spencer

2016 Statecraft and expansionary dynamics: A Virú outpost at Huaca Prieta, Chicama Valley, Peru, PNAS 113 (41), E6016-E6025. https://doi.org/10.1073/pnas.1609972113

Prieto, G.

2018 Informe anual. Programa Arqueológico Huanchaco 2016-2017, Municipalidad Distrital de Huanchaco, Huanchaco.

Roux, V.

2007 Ethnoarchaeology: A non-historical science of reference necessary for interpreting the past, Journal of Archaeological Method and Theory 14 (2), 153-178. https://doi.org/10.1007/s10816-007-9030-8

2016 Des céramiques et des hommes, Presses Universitaires de Paris Ouest, Paris.

Roux, V. y M-A. Courty

2005 Identifying social entities at a macro-regional level: chalcolithic ceramics of south Levant as a case study, en: D. Bosquet, A. Livingstone-Smith y R. Martineau (eds.), Pottery Manufacturing Processes: Reconstruction and Interpretation, 210-214, Archaeopress, Oxford.

Ramón, G.

2011 The swallow potters: seasonally migratory styles in the Andes, en: S. Scarcella (ed.), Archaeological ceramics: A review of current research, 160-175, Archaeopress, BAR International Series 2193, Oxford.

2013 Los alfareros golondrinos, productores itinerantes en los Andes, Instituto Francés de Estudios Andinos/ Sequilao Editores, Lima.

Shimada, I. y A. Maguiña

1994 Nueva visión sobre la cultura gallinazo y su relación con la cultura moche, en: Santiago Uceda and Elías Mujica (eds.), Moche: Propuestas y perspectivas, 31-58, Travaux de l'Institut Français d'Études Andines 79, Lima: Universidad Nacional de La Libertad/Instituto Francés de Estudios Andinos. https://doi. org/10.4000/books.ifea.2380

Strong, W. y C. Evans

1952 Cultural Stratigraphy in the Virú Valley, Northern Peru, The Formative and Florescent Epochs, Columbia University Press, New York. https://doi.org/10.7312/stro90798 
Tantaleán, $\mathrm{H}$.

2016 Una historia de la arqueología peruana, Instituto de Estudios Peruanos/Universidad San Francisco de Quito, Quito.

Uceda, $S$.

2016 La presencia foránea en el complejo Huacas del Sol y de la Luna: relaciones políticas y sociales de los Mochicas, en: C. Pardo y J. Rucabado (eds.), Moche y sus vecinos. Reconstruyendo identidades, 68-99, Asociación Museo de Arte de Lima, Lima.

\section{Uceda S., H. Gayoso y N. Gamarra}

2009 The Gallinazo at Moche: Style or culture?, en: J-F. Millaire y M. Morlion (eds.), Gallinazo. An early cultural tradition on the Peruvian North Coast, 105-124, Cotsen Institute of Archaeological Press, University of California, Los Angeles.

Willey, G.

1953 Prehistoric Settlement Patterns in the Virú Valley, Smithsonian Institution, Bureau of American Ethnology, Bulletin 155, Washington, D.C.

Recibido: 31/01/2019

Aceptado: 31/07/2019 